\title{
Fuzzy and Neural Controllers for a Pneumatic Actuator
}

\author{
Tiberiu Vesselenyi, Simona Dziţac, Ioan Dziţac, Mişu-Jan Manolescu
}

\begin{abstract}
There is a great diversity of ways to use fuzzy inference in robot control systems, either in the place where it is applied in the control scheme or in the form or type of inference algorithms used. On the other hand, artificial neural networks ability to simulate nonlinear systems is used in different researches in order to develop automated control systems of industrial processes. In these applications of neural networks, there are two important steps: system identification (development of neural process model) and development of control (definition of neural control structure). In this paper we present some modelling applications, which uses fuzzy and neural controllers, developed on a pneumatic actuator containing a force and a position sensor, which can be used for robotic grinding operations. Following the simulation one of the algorithms was tested on an experimental setup. The paper also presents the development of a NARMA-L2 neural controller for a pneumatic actuator using position feedback. The structure had been trained and validated, obtaining good results.
\end{abstract}

Keywords: fuzzy control, neural control, force-position feedback, pneumatic actuator.

\section{Introduction}

There is a great diversity in which fuzzy inference and neural networks can be used in robotics operation control either in the place it has in the control scheme or in the type of fuzzy or neural controller. From the studied references the conclusion can be drawn that fuzzy inference is used (among others) in trajectory generation [3], robot model design [2], instead of P.I.D. controllers [4] or in combination with these [6]. A detailed presentation of general purpose fuzzy controllers is given in [5]. In the same work, it is shown that there can be made fuzzy controllers similar to classical ones (quasi - P.I.D.). In other researches the importance of parameter adjustment is emphasized and also that fuzzy controllers can be adjusted more easily [6]. Due to the fact that a large part of fuzzy inference systems had been implemented on heuristic basis (usually the membership functions are chosen upon the educated guess of specialists) there is no guarantee of a reliable operation or stability of the system in unforeseen conditions. Due to this, experimental tests must be considered. A great number of researches in this field have as goal the development of methodologies of synthesis and analysis of fuzzy inference systems, in the field of robotics [2], [4] or in the larger field of control systems [5] (i.e. study of stability of fuzzy controllers).

Also there are works regarding the elaboration of fuzzy models of robots (used in direct and inverse kinematics [3] or in inverse dynamics [1], which can replace analytical models, and shorten the computing times. Many researches try a systematic approach of fuzzy systems design (development of a design methodology), which can eliminate the subjectivity in choosing the membership functions and rule sets, as in [2], in which a clear method is presented for a rigorous selection of fuzzy inference parameters.

In order to develop a model and test fuzzy and neural control in the design phase an adequate programming environment must be selected. For this purpose we had chosen the MATLAB programming environment, because it offers predefined functions to develop fuzzy and neural control systems. These functions are linked to extern modules like the "inference system" and the "fuzzy engine", and the SIMULINK module can also use these functions. User applications can be linked to these modules using the predefined functions.

The typical base structure of fuzzy systems develop a model which make the correspondence: 
- crisp value - input membership functions - inference rules -

- output characteristics - output membership functions - crisp output value.

Also, a typical fuzzy inference system, supposes a user defined set of parameters which try to encrypt the model's variables characteristics. If instead the development of a process model is wanted for which certain experimental input-output data sets exists, the fuzzy system parameters can be automatically generated that is the system identification can be done. In this case the identification strategy can be a neural-fuzzy approach, which has at its base acquiring knowledge from the presented data set in order to generate membership function parameters. In the MATLAB environment the adjustment of these parameters can be done with a module which works similar to a neural network named ANFIS (Adaptive Neural Fuzzy Inference System). As teaching algorithm error back propagation is used and the optimization is made by a gradient method, followed by error minimization (by the quadratic sum method).

In [10], a methodology is presented for designing an adaptive fuzzy logic controller. "The neurofuzzy controller is first trained using data from an approximate analytical model of a cellular network then the controller is fine tuned and adapted to the unique cell dwell time and call holding time distributions of a particular cell in the network".

The ability of neural networks to simulate non-linear systems, is used in some researches [13], in order to develop industrial processes control systems. When using neural networks in controlling processes there have to be two steps: system identification (development of process neural model) and control design (development of neural control system).

In the system identification step, the neural model of the controlled process is developed and in the second step this model is used to obtain the neural net that will control the process. The training of process neural model is made "offline" (or "batch processing"), but the training and optimization of neural control must be made "online" using training data sets.

A chaos search immune algorithm is proposed in [12] by integrating the chaos optimization algorithm and the clonal selection algorithm: "first, optimization variables are expressed by chaotic variables through solution space transformation. Then, taking advantages of the ergodic and stochastic properties of chaotic variables, a chaos search is performed in the neighborhoods of high affinity antibodies to exploit local solution space, and the motion of the chaotic variables in their ergodic space is used to explore the whole solution space. Furthermore, a generalized radial basis function neuro-fuzzy controller [...] is constructed and designed automatically".

\section{Simulation of pneumatic system with fuzzy controller}

\subsection{General considerations}

The general scheme of the automated grinding system is presented in figure 1. This system is used to grind metal probes for microscope observations.

The "Command module" represents a programmable computing unit on which the control algorithm is running (fuzzy or neural controller - in this case a Pentium IV PC) and it has the possibility to transmit signals to the execution unit and to receive data from sensors (by means of a DAQ card with analogical and digital channels).

The "Execution module" contains the execution elements, which are simple and proportional electro - valves, "Force sensor" and "Position sensor" - are the sensor used to generate the feedback signals.

The pneumatic setup of the force-position feedback system (FPFS) is shown in figure 3. For the position feedback (PFS) system the force sensor is missing.

In the position feedback case the system has to move the probe, approaching the grinding surface to an approximate distance, and then to move it with smaller speed to touch the surface. 


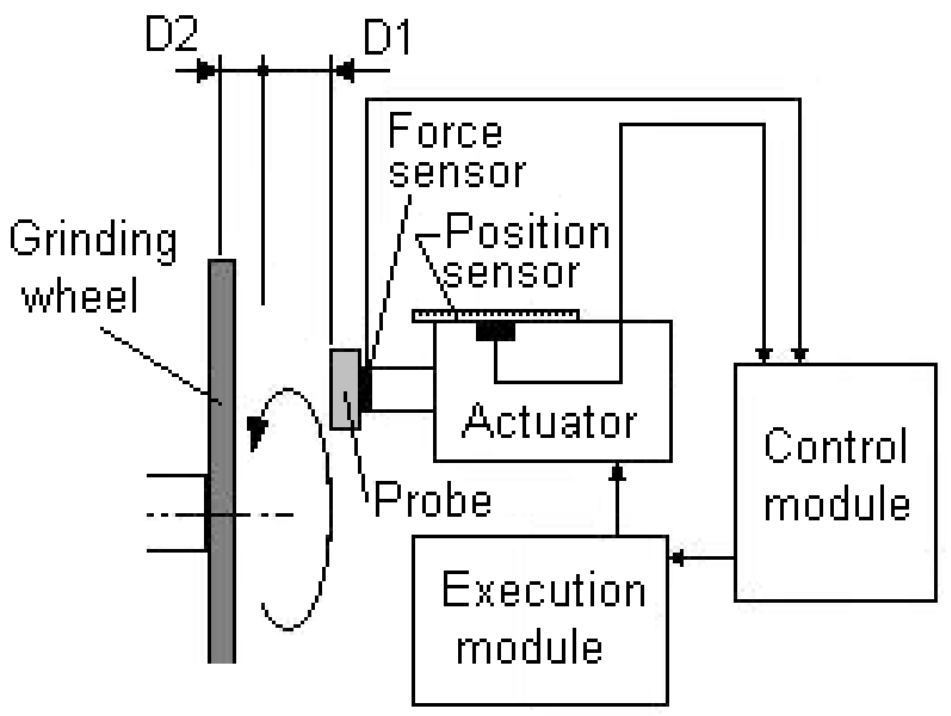

Figure 1: Scheme of automated metal probe grinding system

In the force - position feedback case the system has to fulfil the objectives represented in diagram presented in figure 2, that is to move the probe near the grinding wheel surface and then to move it with reduced speed until the force reaches a reference value and then again to maintain the probe pushed on the surface with the reference force. Above described strategies can be graphically expressed in the diagram shown in figure 3.

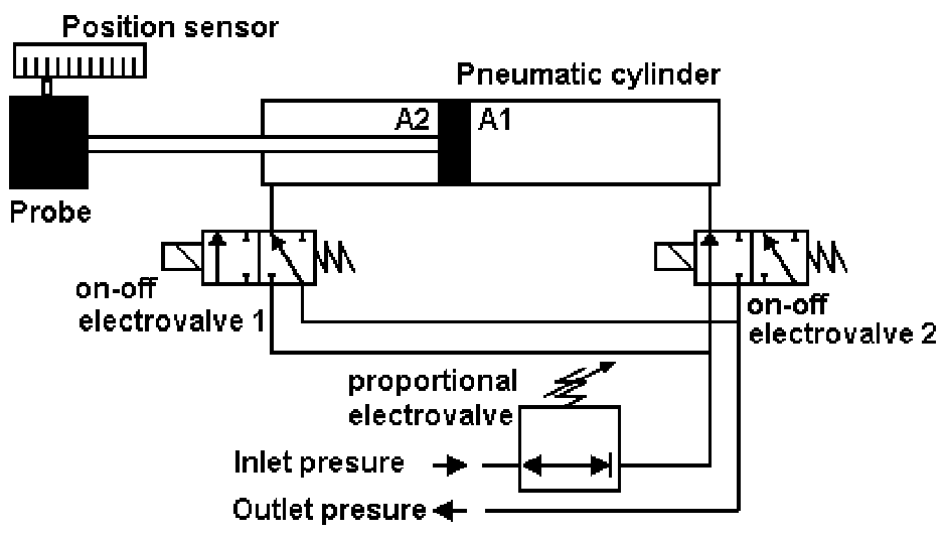

Figure 2: Scheme of pneumatic setup

For the fuzzy FPFS case (the complete case) there have to be defined:

- two input linguistic variables "Position error" and "Forces error" and one output linguistic variable named "Control value".

- position reference is given by the superior limit of the uncertainty domain D2;

- the final control will be made by the "Force" variable;

- for position there are two ranges which must be defined (Big and Small) and for the force seven ranges (Big negative, Medium negative, Small negative, Zero, Big positive, Medium positive, Small positive). 


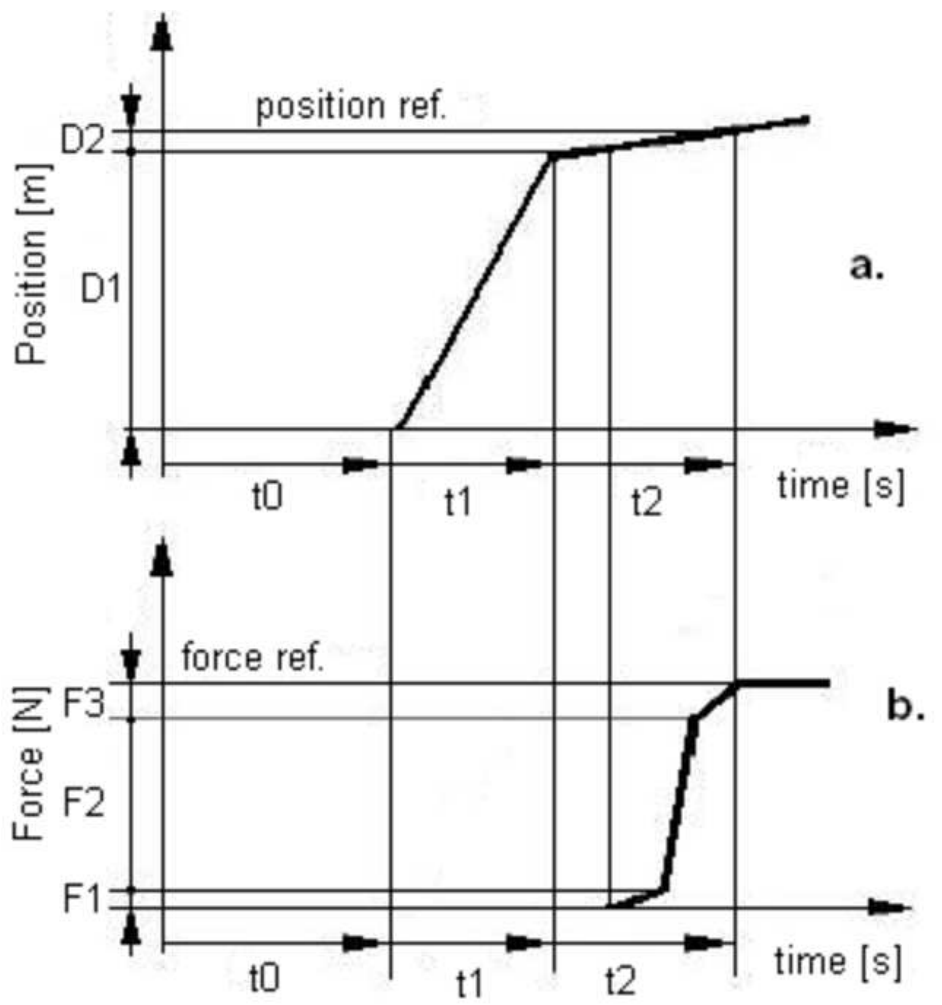

Figure 3: Functional diagrams for position (a) and force (b) control

\subsection{Fuzzy position feedback system}

The model for fuzzy PFS is presented in figure 4. In this case the system is used without force reaction. This model has been made only as a preliminary study.

In figure 5 the systems inference diagrams are shown:

- the gaussian input membership functions represents 5 linguistic ranges of the "Position error";

- the output triangular membership functions represent also 5 linguistic ranges of the output variable "Control value"; deffuzyfication is made by a "centroid" function.

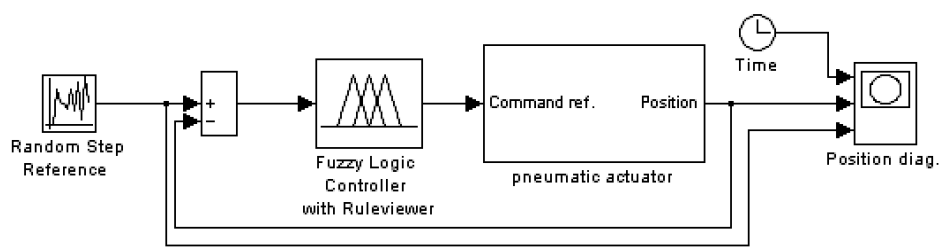

Figure 4: Fuzzy PFS model

Results of simulation is given in figure 6 for some random step reference values. We can observe how the actual controlled position is following the reference position. The delays are relatively large but considering that the pneumatic system is acting similar to a damper, this range of delay is acceptable.

\subsection{Fuzzy force - position feedback system}

The fuzzy FPFS is presented in figure 7. 

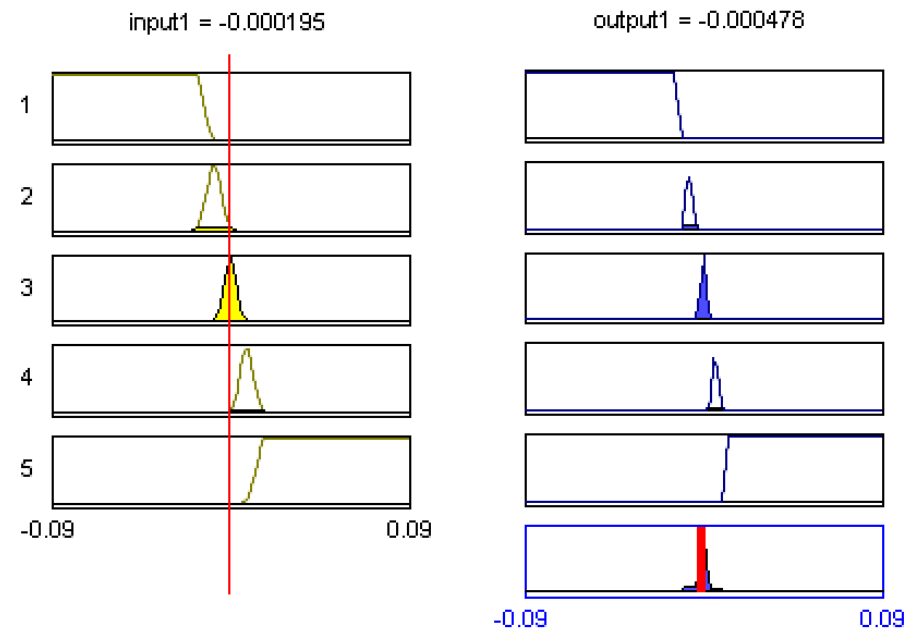

Figure 5: Inference diagram of the fuzzy PFS model

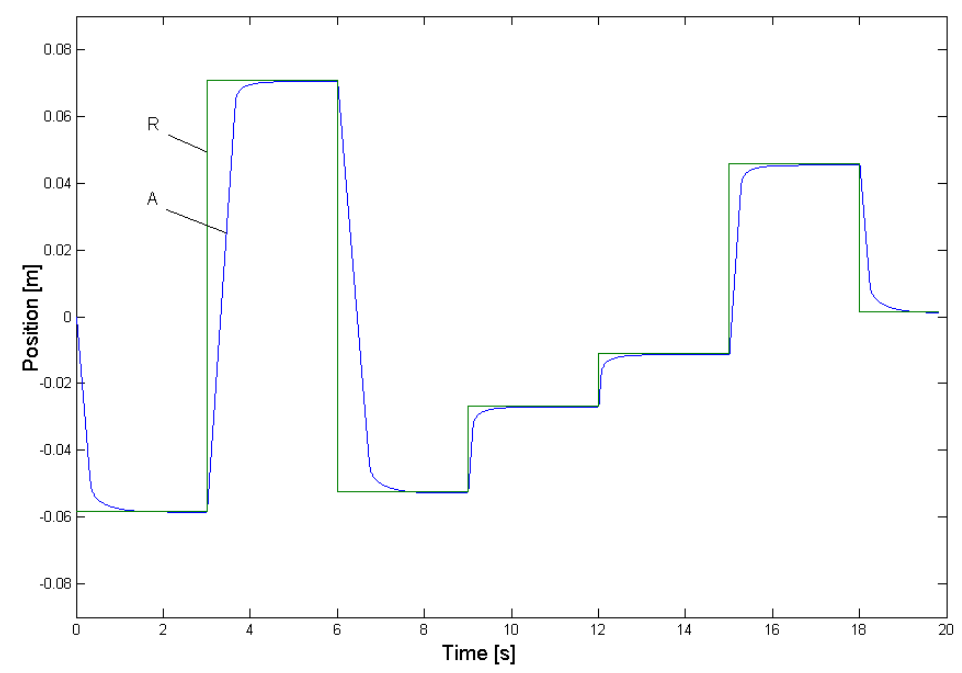

Figure 6: Reference (R) and actual (A) signals diagram resulted from system simulation (fuzzy PFS case)

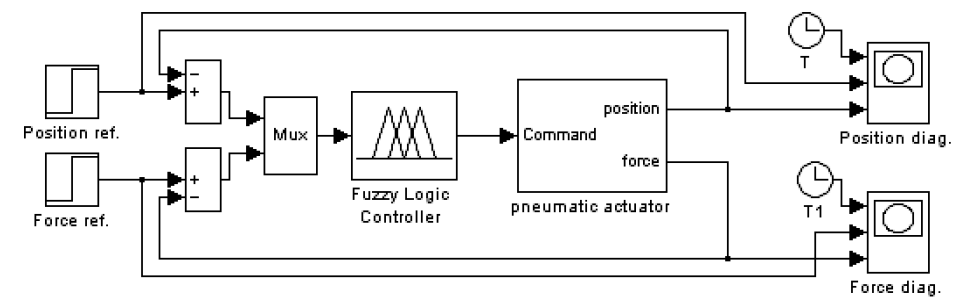

Figure 7: Fuzzy FPFS model 
For both inputs (as for "Force error" as for "Position error") the membership functions are "gaussian" type and for output membership function the "triangular" type had been used. Simulation results for step type references are given in figures 8 and 9.

Analyzing the result diagram we can conclude that the control is working correctly.

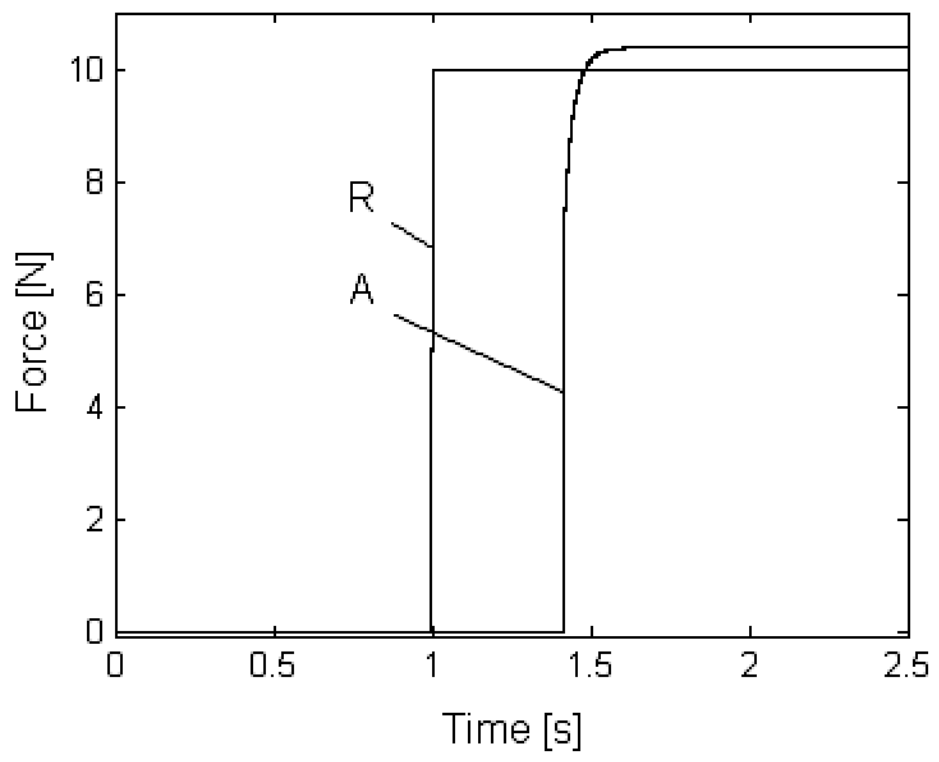

Figure 8: Reference and response force values

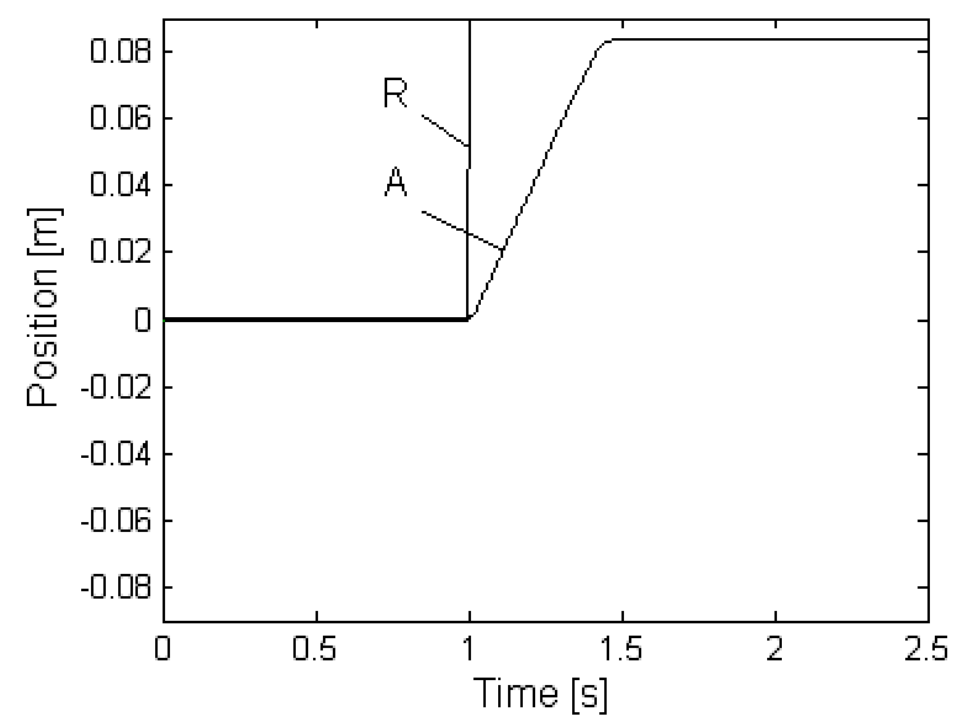

Figure 9: Reference and response position values

\section{Experimental setup for fuzzy FPFS}

The experimental study in order to test fuzzy FPFS operation is shown in figure 10. The base idea of this concept was the use of a PCI6023E DAQ card (from National Instruments), for which there are predefined acquisition functions in the MATLAB "Data Acquisition Toolbox". This fact makes possible the 
use of data acquisition, fuzzy inference engine and command signal generation from the same program. Even the designed system gives birth to considerable delays in comparison with a true real time system (which would use xPCTarget modules), it is good enough for testing of controllers accuracy.

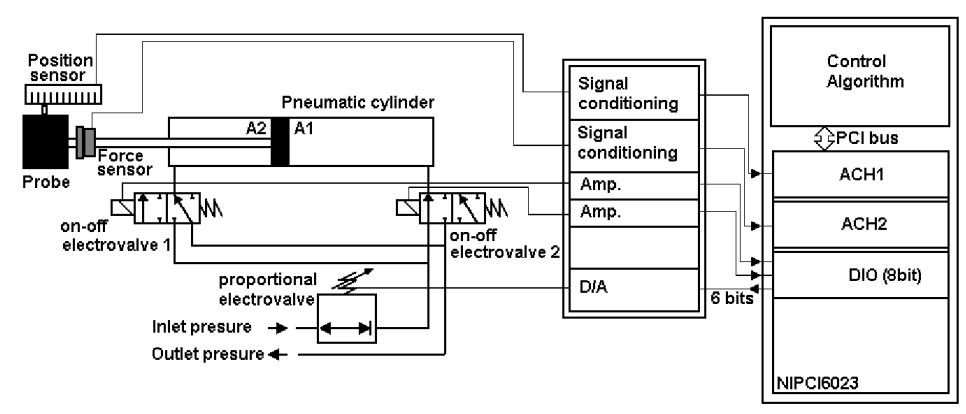

Figure 10: Experimental setup scheme

The DAQ card has 8 analogical input channels (ACH1...8) on 12 bits, from which $\mathrm{ACH} 1$ and $\mathrm{ACH} 2$. were used.

A major disadvantage of PCI6023 DAQ card is the absence of analog output, which would be used to command the pressure regulator proportional electro-valve (figure 10). This issue was solved by using 6 out of 8 digital channels of the digital I/O port (DIO in figure 10) and a D/A (digital to analog) converter. So we can obtain 64 values of pressure for the 6 bits available, which suffice for the experiments. We cannot use al the 8 cannels of the DIO port because 2 channels must be used to command the 2 on-off electro-valves used to change the piston's movement direction.

\section{Neural position feedback system}

In the case of neural PFS there must be two steps to complete: system identification (development of process neural model) and control design (development of neural control system) [3].

In the system identification step, the neural model of the controlled process is developed and in the second step this model is used to obtain the neural net that will control the process.

\subsection{Identification step}

In the identification step a convenient structure for the process model must be found and then the neural network will be trained in order to obtain the value of weights, using training data sets. A largely used standard structure, representative for nonlinear discrete systems is NARMA (Nonlinear AutoRegressive - Moving Average) [3], given by the relation:

$$
y(k+d)=N[y(k), y(k-1), \ldots, y(k-n+1), u(k), u(k-1), \ldots, u(k-n+1)]
$$

in which: $u(k)$ is the system's input and $y(k)$ is the output. In order to identify the process, the network will be trained with the non-linear function $N$.

\subsection{Control step}

If the goal of the system is to follow a reference trajectory, $y(k+d)=y_{r}(k+d)$, a non-linear controller will have to be developed:

$$
u(k)=G\left[y(k), y(k-1), \ldots, y(k-n+1), y_{r}(k+d), u(k-1), \ldots, u(k-m+1)\right]
$$


In order to generate the function $G$, which minimizes the quadratic mean error, a dynamic backpropagation learning algorithm should be used which is hard to implement and very slow. That is the reason why some approximate models are usually used.

Such an approximate model is given by relation:

$$
\begin{aligned}
y(k+d)= & f[y(k), y(k-1), \ldots, y(k-n+1), u(k-1), \ldots, u(k-m+1)] \\
& +g[y(k), y(k-1), \ldots, y(k-n+1), u(k-1), \ldots, u(k-m+1)] \cdot u(k)
\end{aligned}
$$

This model is in a form in which the input $\mathrm{u}(\mathrm{k})$ is not contained in the non-linear term and if $y(k+d)=y_{r}(k+d)$, it can then be written that:

$$
u(k)=\frac{y_{r}(k+d)-f[y(k), y(k-1), \ldots, y(k-n+1), u(k-1), \ldots, u(k-n+1)]}{g[y(k), y(k-1), \ldots, y(k-n+1), u(k-1), \ldots, u(k-n+1)]}
$$

In this form it is necessary to find the input values $u(k)$, based on the output in the same step $y(k)$, which is inconvenient and is better to use the form:

$$
\begin{aligned}
y(k+d)= & f[y(k), y(k-1), \ldots, y(k-n+1), u(k), u(k-1), \ldots, u(k-n+1)] \\
& +g[y(k), y(k-1), \ldots, y(k-n+1), u(k), \ldots, u(k-n+1)] \cdot u(k+1)
\end{aligned}
$$

for $d \geq 2$.

The structure of the process model neural network neural network is given in figure 11 .

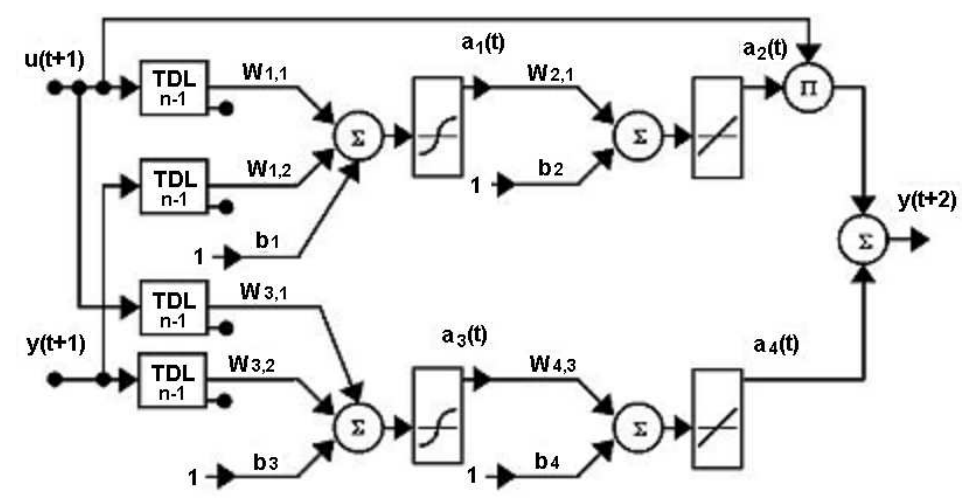

Figure 11: Process neural model based on relation (5)

The controller expression from relation 5, will be:

for $d \geq 2$.

$$
u(k+1)=\frac{y_{r}(k+d)-f[y(k), \ldots, y(k-n+1), u(k), \ldots, u(k-n+1)]}{g[y(k), \ldots, y(k-n+1), u(k), \ldots, u(k-n+1)]}
$$

In figure 12 is presented the control scheme in which yr is generated by the neural model ("reference model").

In this case the controller has to make only a few computations and the neural model can be trained off-line. The method can be applied in industrial robot control, but it was not tested yet with pneumatic actuators. The real advantage of using this kind of controllers for industrial robots, would be the use of parallel system for each axes of the robot.

As support of computational implementation of the simulation the SIMULINK module of MATLAB was used. SIMULINK, contains predefined blocks to generate the neural model as well a GUI for parameter settings, training and validation of the controller. 


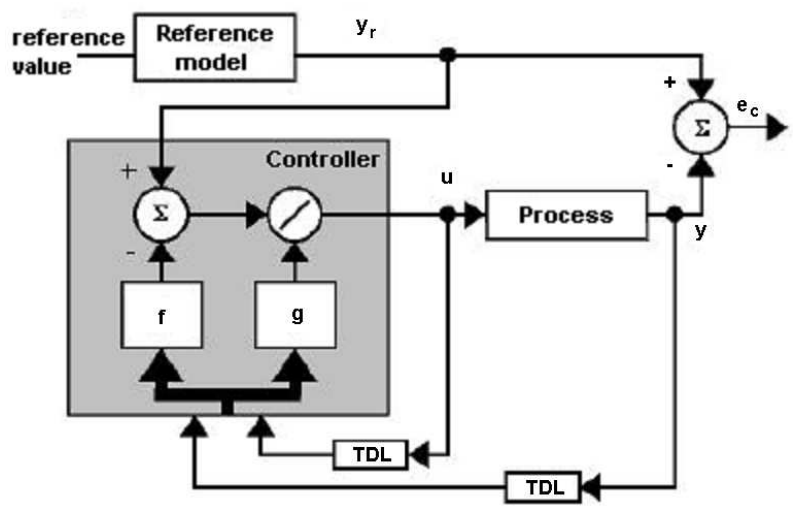

Figure 12: Control scheme designed on the relation (6)

\subsection{Neural position feedback system simulation}

For this case a proportional valve and two on-off electro-valves have been used. The pneumatic design scheme is the same as it was presented in figure 2, only the controller structure is different.

This model is very complex and even if the PC used for simulation (Intel Pentium IV 2GHz), has a significant computing power training cycles are very long (about hundreds of minutes). That is why we have used the simplified model of the process shown in figure 13. The simplification is made by neglecting the air compressibility terms and the elimination of air from cylinders inactive chamber.

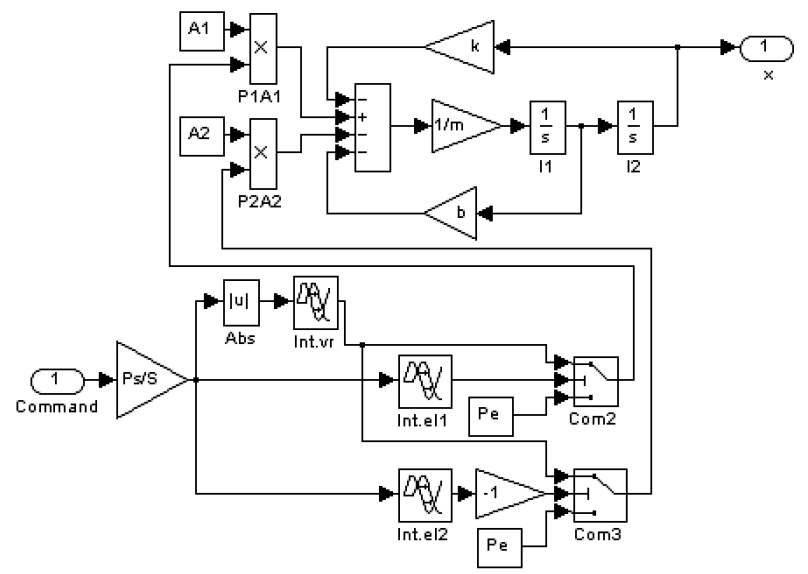

Figure 13: Simplified process scheme

The presented model is then integrated with the controller model given the final version shown in figure 14 .

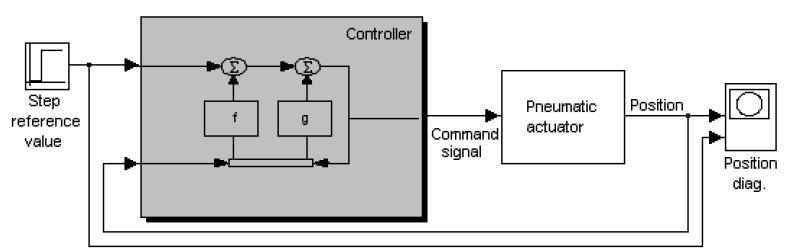

Figure 14: Process with controller scheme 
After model design, the training, validation and test data sets were generated with the help of process model. On this basis the neural model of process and controller were obtained. The training error as function of training epochs is presented in figure 15.

A number of 3000 data in 300 epochs were used as training parameters. As it can be seen from the diagram the convergence of the network is good.

Simulations were carried out for different reference values for position from 0.025 to 0.06 . In figure 16 a sample of obtained response is shown for position reference value of 0.042 .

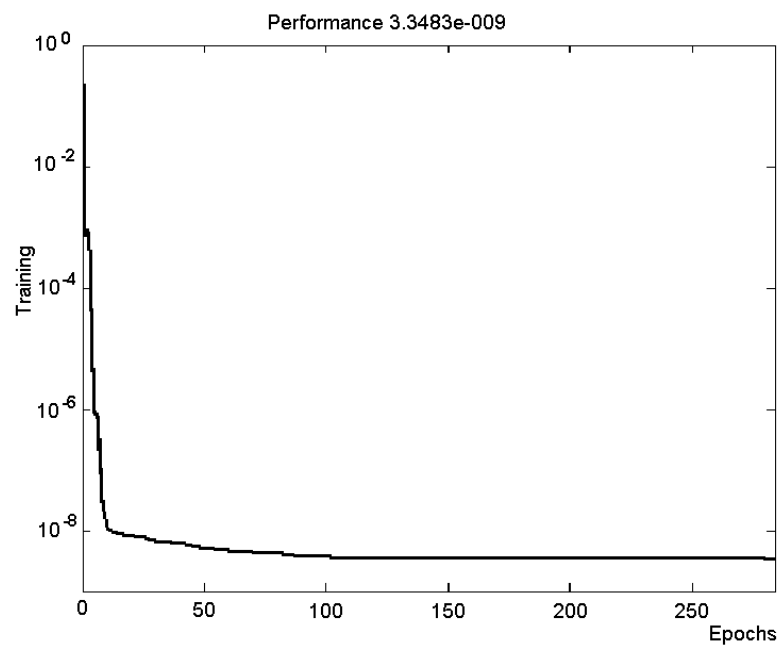

Figure 15: Training of the neural network

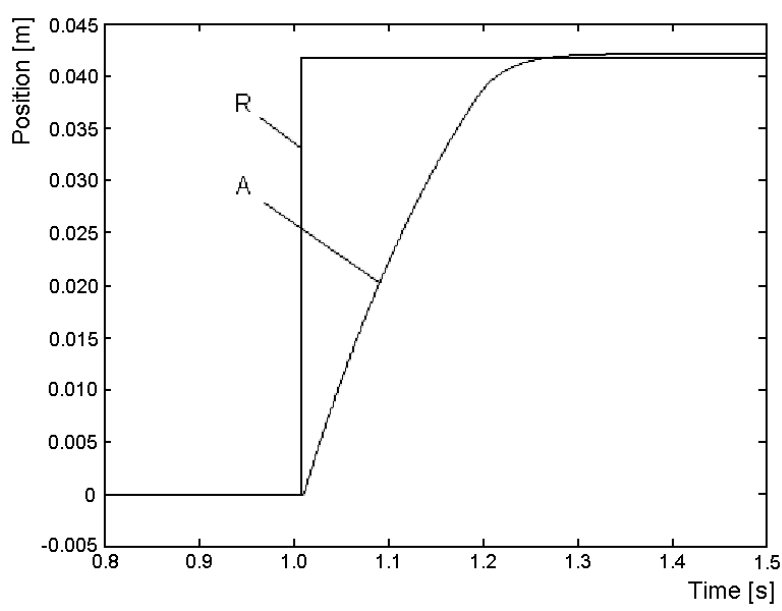

Figure 16: Reference (R) and actual (A)value of simulation results

\section{Results and conclusions}

The structure of the NARMA-L2 neural controller for a pneumatic actuator has been trained and validated, obtaining good results.

After the analysis of simulation results and the experimental system operation it can be said that the fuzzy controller is working in a proper manner for this application but needs further adjustments in order to increase the robustness of the control. 
In actual operation there are unwanted effects as:

- position control error is constant, but the actual position remains under the reference value with about $6 \%$ for a value of reference from 6 to 9 ;

- the uncertainty domain in which the probe can meet the grinding wheel and the domain in which the control is acceptable is small (about $0.003 \mathrm{~m}$ ), but in practice this is about 0.005 to $0.006 \mathrm{~m}$;

- the force control error is increasing with the increase of reference, limiting the application domain in which the error is still acceptable.

Although in some domains the control is acceptable further adjustments of the control parameters are needed.

In the study of neural controller simulation it resulted that the overshoot error of the system was only of 3\%. In this case further studies must be carried out to implement the neural controller for the FPFS case and to test it experimentally.

\section{Bibliography}

[1] Amann P., Perronne J.,M., Gissinger G.,L., Frank P., M., Identification of fuzzy relational models for fault detection, Control Engineering Practice 9, 555, 2001.

[2] Emami M.R., Goldenberg A.A., Burhan T.R., Systematic design and analysis of fuzzy-logic control and application to robotics, Part I. Modeling, Robotics and Autonomous Systems 33, pp. 65-88, 2000 .

[3] Jang, R., MATLAB - Fuzzy Toolbox - The MathWorks, Inc. Revision: 1.12, Date: 2000, 15.

[4] Novakovic, B., Scap, D., Novakovic D., An analytic approach to fuzzy robot control synthesis, Engineering Applications of Artificial Intelligence 13, pp. 71-83, 2000.

[5] Preitl, St., Precup, E., Introducerea în conducerea fuzzy a proceselor, Ed. Tehnicã, Bucuresti, 1997.

[6] Reznik L., Ghanayem O., Bourmistrov A., PID plus fuzzy controller structures as a design base for industrial applications, Engineering Applications of Artificial Intelligence 13, pp. 419-430, 2000.

[7] Vesselenyi T., Automated flexible cell for microstructure recognition, $\mathrm{PhD}$ Thesis, Universitatea "Politehnica" Timisoara, 2005.

[8] M.M., Chen, J.A., Fairwather, S.A., Green, EDUMECH. Mechatronic Instructional Systems. Case Study: Pneumatics Systems, Production of Shandor Motion Systems, Inc., 1999.

[9] Harbick K., Sukhatme S., Speed Control of a Pneumatic Monopod using a Neural Network, www.harbick-ann.com, 2002.

[10] Raad R., Raad I., Neuro-Fuzzy Admission Control in Cellular Networks. Communication systems, (10th IEEE Singapore International Conference on Communication systems.), pp. 1-7, 2006.

[11] Wenmei H., Yong Y., Yali T., Adaptive neuron control based on predictive model in pneumatic servo system, 2002.

[12] Zuo X.Q., Fan Y.S., A chaos search immune algorithm with its application to neuro-fuzzy controller design. Chaos, Solitons \& Fractals, Vol. 30, Issue 1, pp. 94-109, 2006. 
[13] Zhang J., Knoll A., Schmidt R., A neuro-fuzzy control model for fine-positioning of manipulators, Robotics and Autonomous Systems, 32, pp. 101-113, 2000.

Tiberiu Vesselenyi

University of Oradea

Universităţii St. 1, 410087, Oradea, Romania tvesselenyi@yahoo.co.uk

Simona Dziţac

University of Oradea

Universităţii St. 1, 410087, Oradea, Romania sdzitac@rdslink.ro

Ioan Dziţac Department of Economic Informatics

Agora University of Oradea

Piaţa Tineretului 8, Oradea 410526, Romania idzitac@univagora.ro

Mişu-Jan Manolescu Agora University

Piaţa Tineretului 8, 410526 Oradea, Romania rectorat@univagora.ro

Received: February, 14, 2007 

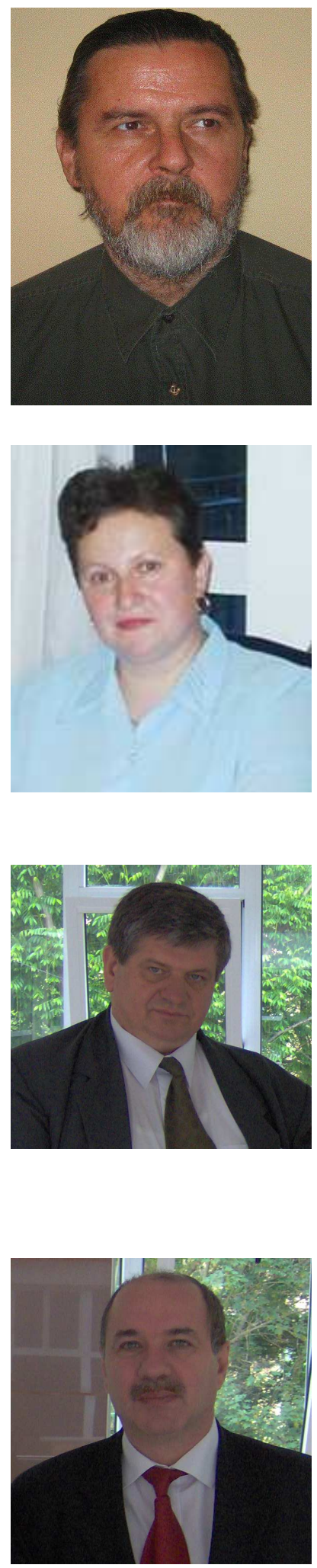

Tiberiu Vesselenyi was born in Oradea, Romania in 1957, he finished the University "Politehnica" from Timişoara in 1983. From 1983 to 1991 he worked at a machine building company in Oradea as designer and CNC programmer. From 1991 till 1994 he was a research engineer at the "Geothermal Energy Research Center" in Oradea and from 1994 till today is assoc. prof. at the University Of Oradea, where he teaches robot and CNC programming. He had earned a $\mathrm{PhD}$ in robotics at the University "Politehnica" at Timişoara. He had published over 150 papers in national and international conferences and journals, and is author or coo - author of 4 books.

Simona Dziţac received B.Sc. (2000) and M. Sc. (2001) in Mathematics-Physics, B.Sc. (2005) and M. Sc. (2007) in Energy Engineering from University of Oradea and B.Sc. in Economic Informatics (2007) from University of Craiova, Romania. At this moment, she is PhD student in Energy Engineering field and researcher at University of Oradea. Her current research interests include Reliability, Applied Mathematics and Computer Science in Engineering fields. She published 4 books and 38 scientific papers in journals and conferences proceedings.

Ioan Dziţac received M. Sc. in Mathematics (1977) and Ph. D in Information Sc. (2002) from "Babes-Bolyai" University of Cluj-Napoca. At this moment, he is associate professor and head of Economic Informatics Department at AGORA University, Oradea, Romania. His current research interests include different aspects of Parallel and Distributed Computing, Applied Mathematics and Economic Informatics. He has edited 4 conference proceedings, published 12 books and 47 scientific papers in journals and conferences proceedings. He was member of the Program Committee of 18 international conferences.

Mişu-Jan Manolescu received M. Sc in Electro-mechanics (1984) from the University of Craiova, Ph. D in Microwave (1994) from the University of Oradea, and Ph. D in Human Resources Management (2000) from the University of Craiova. Now, he is professor and president of AGORA University, Oradea, Romania. His current research interests include different aspects of Knowledge Management, and Knowledge Engineering. He has edited 4 conference proceedings, published 9 books and 63 scientific papers in journals and conferences proceedings. He has been member of Program Committee of the 5 international conferences. 